\title{
Analysis of Passive Filters for High Power Three-level Rectifiers.
}

\author{
Alex-Sander A. Luiz, Braz J. Cardoso Filho \\ Centro Federal Eduacação Tecnológica de Minas Gerais, \\ Depto. de Engenharia Elétrica, B. Horizonte - MG, BRAZIL \\ asal@des.cefetmg.br,
}

\begin{abstract}
This paper studies passive filters for harmonic reduction in high power rectifiers. The paper presents some of the most important configurations of high power filter employed in medium voltage applications. The paper as well includes the ideal mathematic equations and models for those filters, and then compares the filter performances. In a very broad sense, the addition of some resonant harmonic traps to a conventional third order filter results in good filter adaptation to PWM strategies. The filter evaluation highlights this fact. Simulation of a high power three-level Neutral Point Clamped-NPC rectifier also confirms those ideas. Results comprise a high quality waveform under low switching frequency with reasonable converter operation performance. Experimental results for Selective Harmonic Elimination are included.
\end{abstract}

\section{INTRODUCTION}

PWM rectifier operation at ac mains generates undesired harmonic content. Some of the problems addressed to those PWM harmonics are voltage and current distortions, overheating of reactive elements (capacitances and inductances), global power factor deterioration and electromagnetic interference (EMI). For many years, passive filters have been employed for harmonic reduction at the input of different structures [1]-[3]. However, the filter requirements for high power converters are quite different from those of the low power rectifiers. This is mainly related to discrepancies in the semiconductor technologies of the power devices, which limit the maximum converter switching frequency. In the last case, the high frequency modulation sweeps the harmonic content to the high frequency regions where the passive filter exhibits superior frequency response and attenuation ratio. Oppositely high power rectifiers, whose switching frequencies are limited from about 500 to $1000 \mathrm{~Hz}$, produce harmonics near to base (fundamental) frequency with practically all modulation strategies. Owing to a poor low frequency performance, (between the cut-off frequency and less than ten times its value) such low frequency harmonics are of difficult elimination. The filter must attenuate them properly in order to assure a good converter operation. Besides, the filter has to be able to deal with them to since they can also excite filter/system resonances.

The work analyzes some passive filters for high power rectifiers. For this purpose, paper brings six sections. The first section introduces the problem of employing passive filters in high power medium voltage applications. Section II presents some of the most important filter configuration in literature. Section III develops the filter mathematic models. Section IV compares the filter performances, and it reinforces the
Braz J. Cardoso Filho

Universidade Federal de Minas Gerais, Depto. de Engenharia Elétrica, B. Horizonte - MG, BRAZIL cardosob@ufmg.br.

analysis in previous section. Section V presents Selective Harmonic Elimination which is an important modulation technique considering the limited switching frequency in this work. It makes feasible the filter design due to its larger frequency range free of harmonics. Finally, simulations of a high power three-level NPC rectifier, in section VI, results in high quality waveforms, under low switching frequency, with reasonable converter operation performance. The paper also includes experimental results for the modulator and converter.

\section{High POWER FILTERS}

In general passive filters consist of reactive elements in either series or parallel branches according to a given filter configuration or profile (low-pass, high-pass, etc.). Fig. 1 brings some of the most important filter configurations for high power converters in the literature [1]-[4]. They are the first order $L$ filter, the third order $L C L$ filter, the tuned $L C L ' L$ filter and the $L C L$ filter with harmonic traps.

\section{MAthematic EQUATIONS AND FILTER ModEls.}

In order to simplify the analysis this section assumes:

- Ideal reactive elements, (no damping and losses).
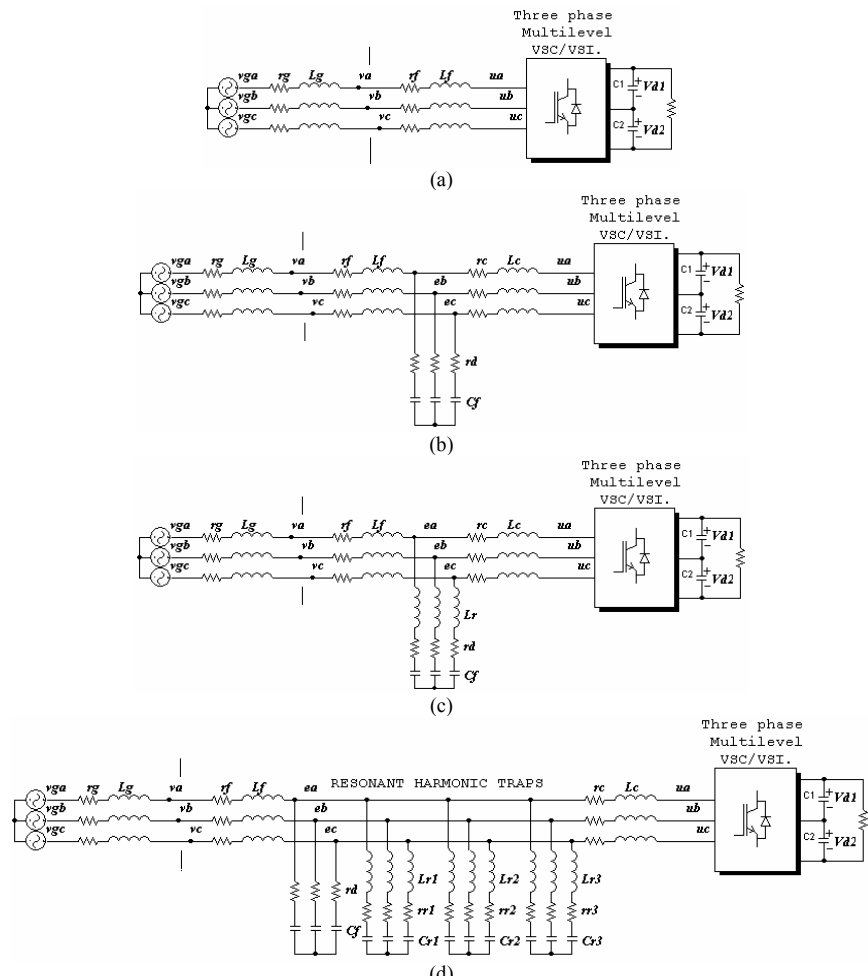

Fig. 1. High power filter configurations a) $L$ filter, b) Third order $L C L$ filter c) Tuned $L C L$ ' $L$ filter and d) $L C L$ filter with harmonic traps. 
- The converter produces all system harmonic content, (the utility grid voltage is purely sinusoidal).

- All voltage distortions at utility grid results exclusively from harmonic currents through the series branch composed by transformer equivalent short-circuit impedance.

A. First order L filter.

Generically the first order $L$ filter consists of a single inductance per phase with a low-pass profile. It is the most common filter configuration for PWM rectifiers. Fig. 2 presents the system equivalent circuit composed by the $L$ filter, (represented by $L_{f}$ and $r_{f}$, corresponding to filter reactive and resistive components), the input insulation transformer, (represented by a purely sinusoidal source $v_{g}$ and the short-circuit impedance elements $L_{g}$ and $r_{g}$ ), and the high power rectifier working as the unique harmonic source in the circuit, (represented by the pulse generator $u$ ).

The equivalent circuit permits to write the following expressions:

$$
\begin{gathered}
v_{g}=r_{t} i_{g}+L_{t} d i_{g} / d t+u \\
v_{g}=r_{g} i_{g}+L_{g} d i_{g} / d t+v
\end{gathered}
$$

where $r_{t}=r_{g}+r_{f}, L_{t}=L_{g}+L_{f} . i_{g}$ and $v$ are respectively the utility grid current and voltage at the point of common coupling - PCC.

Taking the Laplace transforms of (1) and (2):

$$
\begin{aligned}
& v_{g}(s)=\left(r_{t}+s L_{t}\right) i_{g}(s)+u(s) \Rightarrow \\
& i_{g}(s)=\left(v_{g}(s)-u(s)\right) /\left(r_{t}+s L_{t}\right) \\
& v_{g}(s)=\left(r_{g}+s L_{g}\right) i_{g}(s)+v(s) \Rightarrow \\
& v(s)=v_{g}(s)-\left(r_{g}+s L_{g}\right) i_{g}(s)
\end{aligned}
$$

Defining the transfer functions $H_{v}(s)=i_{g} / v$ and $H_{u}(s)=i_{g} / u$ :

$$
i_{g}(s)=H_{v}(s) v_{g}(s)+H_{u}(s) u(s)
$$

$$
v(s)=\left[1-\left(r_{g}+s L_{g}\right) H_{v}(s)\right] v_{g}(s)-\left(r_{g}+s L_{g}\right) H_{u}(s) u(s)
$$

In this case, $H_{v}(s)=-H_{u}(s)=1 /\left(r_{t}+s L_{t}\right)$. Replacing the Laplace operator $s$ with $j n \omega_{1}$ :

$$
H_{v}\left(j n \omega_{1}\right)=-H_{u}\left(j n \omega_{1}\right)=1 /\left(r_{t}+j n \omega_{1} L_{t}\right)
$$

$v\left(j n \omega_{1}\right)=\left[1-\left(r_{g}+j n \omega_{1} L_{g}\right) H_{v}\left(j n \omega_{1}\right)\right] v_{g}\left(j n \omega_{1}\right)-\left(r_{g}+j n \omega_{1} L_{g}\right) H_{u}\left(j n \omega_{1}\right) u\left(j n \omega_{1}\right)$

where $\omega_{1}$ is the fundamental angular frequency of the source $v_{g}$ and converter $u$ voltages and $n$ is the harmonic order.
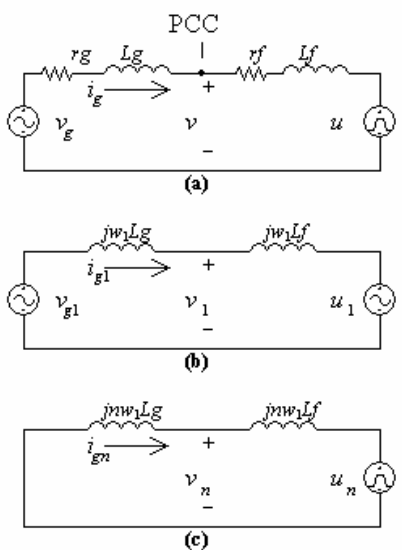

Fig. 2. System model with $L$ filter. a) Single phase equivalent circuit, b) equivalent circuit for the fundamental frequency, c) equivalent circuit for the harmonic frequencies.
Assuming that $\omega_{1} L_{x}$ is in general greater than $r_{x}\{x=g, f$ or $t\}$, it is possible to neglect $r_{x}$ since $n \omega_{1} L_{x}>>r_{x}$. Then:

$$
H_{v}\left(j n \omega_{1}\right)=-H_{u}\left(j n \omega_{1}\right)=1 / j n \omega_{1} L_{t}
$$

$v\left(j n \omega_{1}\right)=\left[1-\left(j n \omega_{1} L_{g}\right) H_{v}\left(j n \omega_{1}\right)\right] v_{g}\left(j n \omega_{1}\right)-\left(j n \omega_{1} L_{g}\right) H_{u}\left(j n \omega_{1}\right) u\left(j n \omega_{1}\right)$

The equivalent circuit of Fig. 2a can be split in two new circuits, one at fundamental frequency $\omega_{1}$ and other at the frequency of $n^{\text {th }}$ harmonic $n \omega_{1}$. Neglecting the transformer harmonic voltage $v_{g},\left\{v_{g n}=0, \forall n \in \mathrm{N} / n \neq 1\right\}, \mathrm{N}$ represents the mathematic set of natural numbers:

$$
\begin{gathered}
i_{g 1}=\left(v_{g 1}-u_{1}\right) / j \omega_{1} L_{t} \\
i_{g n}=-u_{n} / j n \omega_{1} L_{t} \\
v_{1}=\left[1-\left(L_{g} / L_{t}\right)\right] v_{g 1}+\left(L_{g} / L_{t}\right) u_{1} \\
v_{n}=\left(L_{g} / L_{t}\right) u_{n}
\end{gathered}
$$

The rms value of grid current $I_{d i s}$ and voltage $V_{\text {dis }}$ distortions defined in [5] are:

$$
\begin{gathered}
I_{d i s}=\sqrt{\sum_{n=2}^{n_{\max }} i_{g n}^{2}}=\left(\frac{1}{\omega_{1} L_{t}}\right) \sqrt{\sum_{n=2}^{n_{\max }}\left(\frac{U_{n}}{n}\right)^{2}} \\
V_{d i s}=\sqrt{\sum_{n=2}^{n_{\max }} v_{n}^{2}}=\left(\frac{L_{g}}{L_{t}}\right) \sqrt{\sum_{n=2}^{n_{\max }} U_{n}^{2}}
\end{gathered}
$$

Here $n_{\max }$ is the highest harmonic order selected to suit a given design specification and $U_{n}$ is rms value of $n^{\text {th }}$ converter harmonic voltage.

The expressions above show that the current harmonic attenuation increases with the harmonic order and the total inductance $\left(L_{t}=L_{g}+L_{f}\right)$. Moreover, the voltage harmonic attenuation depends only on the inductance relation $L_{g} / L_{t}$, (typically, about from 1 to $20 \%$ since the transformer shortcircuit inductance $L_{g}$ is small). Therefore, further improvement in the voltage harmonic attenuation to suit some design specification requires some increment in $L_{f}$. This in general results in bulky and heavy filter elements.

\section{B. Third order LCL filter.}

The $L C L$ filter is an alternative to the first order $L$ filter. It consists of a third order low-pass configuration with reactive elements displaced in "T" as in Fig. 3. Recently, the $L C L$ filter has been exhaustively investigated with PWM rectifiers, [4], [6], and others. From the system equivalent circuit:

$$
\begin{gathered}
v_{g}=r_{t} i_{g}+L_{t} d i_{g} / d t+e \\
e=r_{c} i+L_{c} d i / d t+u \\
v_{g}=r_{g} i_{g}+L_{g} d i_{g} / d t+v \\
i_{g}=i_{f}+i \\
i_{f}=C_{f} d e / d t
\end{gathered}
$$

where $L_{c}$ and $r_{c}$ are respectively the converter side series branch inductance and resistance, $i$ is current through this series branch, $C_{f}$ is the parallel branch capacitor, and $e$ is voltage across this branch. Then taking the Laplace transforms of (17)-(21) and assuming the expressions (5) and (6) valid for the $L C L$ filter:

$$
\begin{gathered}
H_{v}(s)=\left(1+s^{2} L_{c} C_{f}\right) / s\left(L_{t}+L_{c}\right)\left(1+s^{2} L_{e q} C_{f}\right) \\
H_{u}(s)=-1 / s\left(L_{t}+L_{c}\right)\left(1+s^{2} L_{e q} C_{f}\right)
\end{gathered}
$$



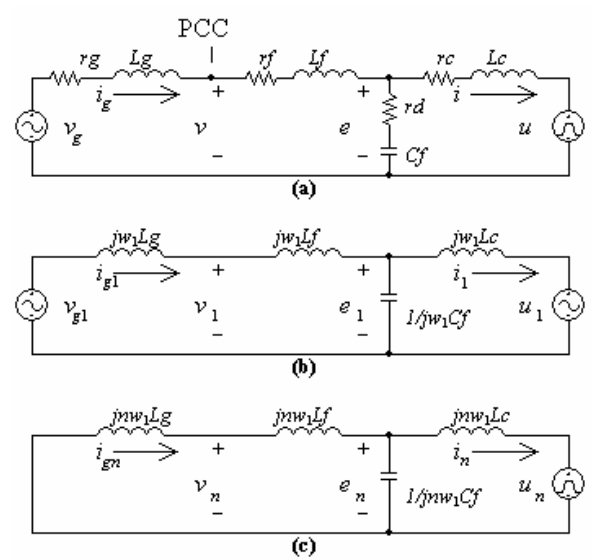

Fig. 3. System model with $L C L$ filter. a) Single phase equivalent circuit, b) equivalent circuit for the fundamental frequency, c) equivalent circuit for the harmonic frequencies.

Here $L_{e q}=L_{t} L_{c} /\left(L_{t}+L_{c}\right)$. For simplification, all resistive and damping elements are neglected. Replacing the Laplace operator $s$ with $j n \omega_{1}$ and splitting the filter equivalent circuit in two new circuits, one at $\omega_{1}$ and other at $n \omega_{1}$ and finally neglecting the transformer harmonic voltage $v_{g n}$ :

$$
\begin{gathered}
i_{g 1}=\frac{\left(1-\omega_{1}^{2} L_{c} C_{f}\right) v_{g 1}-u_{1}}{j \omega_{1}\left(L_{t}+L_{c}\right)\left(1-\omega_{1}^{2} L_{e q} C_{f}\right)} \\
v_{1}=-u_{n} / j n \omega_{1}\left(L_{t}+L_{c}\right)\left(1-n^{2} \omega_{1}^{2} L_{e q} C_{f}\right) \\
\left.1-\frac{L_{g}}{\left(L_{t}+L_{c}\right)} \frac{\left(1-\omega_{1}^{2} L_{c} C_{f}\right)}{\left(1-\omega_{1}^{2} L_{e q} C_{f}\right)}\right] v_{g 1}+\frac{L_{g}}{\left(L_{t}+L_{c}\right)} \frac{1}{\left(1-\omega_{1}^{2} L_{e q} C_{f}\right)} u_{1} \\
v_{n}=\left[L_{g} /\left(L_{t}+L_{c}\right)\right] \cdot\left[u_{n} /\left(1-n^{2} \omega_{1}^{2} L_{e q} C_{f}\right)\right]
\end{gathered}
$$

The rms grid current $I_{\text {dis }}$ and voltage $V_{\text {dis }}$ distortions are:

$$
\begin{gathered}
I_{d i s}=\left[\frac{1}{\omega_{1}\left(L_{t}+L_{c}\right)}\right] \sqrt{\sum_{n=2}^{n_{\max }}\left[\frac{U_{n}}{n\left(1-n^{2} \omega_{1}^{2} L_{e q} C_{f}\right)}\right]^{2}} \\
V_{d i s}=\left(\frac{L_{g}}{L_{t}+L_{c}}\right) \sqrt{\sum_{n=2}^{n_{\max }}\left[\frac{U_{n}}{\left(1-n^{2} \omega_{1}^{2} L_{e q} C_{f}\right)}\right]^{2}}
\end{gathered}
$$

The above equations confirm that $L C L$ improves the $L$ filter performance. Here the product $\omega_{1} L_{e q} C_{f}$, the sum of series branch inductances $\left(L_{t}+L_{c}=L_{g}+L_{f}+L_{c}\right)$ and nearly the third power of $n^{\text {th }}$ harmonic simultaneously affect the current harmonic attenuation. The voltage harmonic attenuation depends on the inductance relation $L_{g} /\left(L_{t}+L_{c}\right)$ and about the square of the product $\omega_{1} L_{e q} C_{f}$, and of the $n^{\text {th }}$ harmonic. The $L C L$ filter introduces inductance $L_{c}$ as a new design element for both current and voltage attenuations, which gives flexibility to the filter design and in general culminates in lighter filter elements, [6].

\section{Tuned LCL'L filter.}

The tuned $L C L^{\prime} L$ filter is another important filter configuration in literature. It is mainly employed in high power uncontrolled rectifiers and consists of reactive elements displaced in " $T$ " but with a resonant parallel branch that can be tuned into a specific converter harmonic frequency as in Fig. 4. The only difference between this and the $L C L$ filter is resonant parallel branch represented by:

$$
e=L_{r} \frac{d i_{f}}{d t}+r_{d} i_{f}+\frac{1}{C_{f}} \int i_{f} d t
$$

In this case, the equations (17)-(20) of the $L C L$ filter are still applicable. Taking the Laplace transforms of them and (30) with resistive elements neglected the transfer functions $H_{v}(s)$ and $H_{u}(s)$ are:

$$
\begin{gathered}
H_{v}(s)=\left[1+s^{2}\left(L_{r}+L_{c}\right) C_{f}\right] / s\left(L_{t}+L_{c}\right)\left[1+s^{2}\left(L_{r}+L_{e q}\right) C_{f}\right] \\
H_{u}(s)=-\left(1+s^{2} L_{r} C_{f}\right) / s\left(L_{t}+L_{c}\right)\left[1+s^{2}\left(L_{r}+L_{e q}\right) C_{f}\right]
\end{gathered}
$$

Replacing the Laplace operator $s$ with $j n \omega_{1}$ and splitting the filter equivalent circuit in two new circuits, one at $\omega_{1}$ and other at $n \omega_{1}$ (with all harmonic voltage $v_{g n}$ neglected):

$$
\begin{aligned}
& i_{g 1}=\frac{\left[1-\omega_{1}^{2}\left(L_{r}+L_{c}\right) C_{f}\right] v_{g 1}-\left(1-\omega_{1}^{2} L_{r} C_{f}\right) u_{1}}{j \omega_{1}\left(L_{t}+L_{c}\right)\left[1-\omega_{1}^{2}\left(L_{r}+L_{e q}\right) C_{f}\right]} \\
& i_{g n}=-\frac{\left(1-n^{2} \omega_{1}^{2} L_{r} C_{f}\right) u_{n}}{j n \omega_{1}\left(L_{t}+L_{c}\right)\left[1-n^{2} \omega_{1}^{2}\left(L_{r}+L_{e q}\right) C_{f}\right]} \\
& v_{1}=\left\{1-\frac{L_{g}\left[1-\omega_{1}^{2}\left(L_{r}+L_{c}\right) C_{f}\right]}{\left(L_{t}+L_{c}\right)}\left[1-\omega_{1}^{2}\left(L_{r}+L_{e q}\right) C_{f}\right]\right\} v_{g 1}+\frac{L_{g}}{\left(L_{t}+L_{c}\right)}\left[\frac{\left(1-\omega_{1}^{2} L_{r} C_{f}\right)}{1-\omega_{1}^{2}\left(L_{r}+L_{e q}\right) C_{f}} u^{u}\right. \\
& v_{n}=\frac{L_{g}}{\left(L_{t}+L_{c}\right)} \frac{\left(1-n^{2} \omega_{1}^{2} L_{r} C_{f}\right) u_{n}}{\left[1-n^{2} \omega_{1}^{2}\left(L_{r}+L_{e q}\right) C_{f}\right.}
\end{aligned}
$$

The rms grid current $I_{d i s}$ and voltage $V_{d i s}$ distortions are:

$$
\begin{gathered}
I_{d i s}=\left[\frac{1}{\omega_{1}\left(L_{t}+L_{c}\right)}\right] \sqrt{\sum_{n=2}^{n_{\max }}\left\{\frac{\left(1-n^{2} \omega_{1}^{2} L_{r} C_{f}\right) U_{n}}{n\left[1-n^{2} \omega_{1}^{2}\left(L_{r}+L_{e q}\right) C_{f}\right]}\right\}^{2}} \\
V_{d i s}=\left(\frac{L_{g}}{L_{t}+L_{c}}\right) \sqrt{\sum_{n=2}^{n_{\max }}\left\{\frac{\left(1-n^{2} \omega_{1}^{2} L_{r} C_{f}\right) U_{n}}{\left[1-n^{2} \omega_{1}^{2}\left(L_{r}+L_{e q}\right) C_{f}\right]}\right\}^{2}}
\end{gathered}
$$

The filter analysis shows that resonant branch has a strong influence on filter behavior. New terms in filter equations such as $\omega_{1}\left(L_{r}+L_{e q}\right) C_{f}$, improve the filter current and voltage harmonic attenuations and also increase the flexibility of filter design. The product $n^{2} \omega_{1}^{2} L_{r} \mathrm{C}_{f}$ as well enhances the harmonic attenuation in low frequency range. On the other hand, the
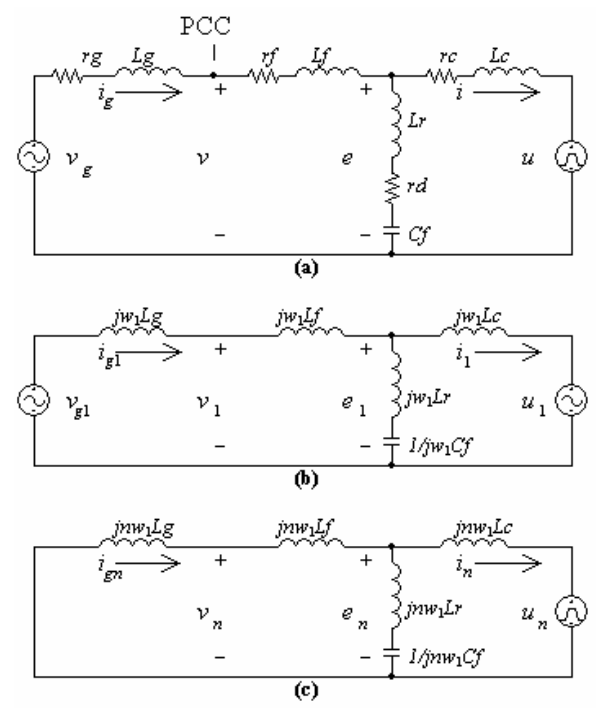

Fig. 4. System model with $L C L^{\prime} L$ filter. a) Single phase equivalent circuit b) equivalent circuit at the fundamental frequency, c) equivalent circuit at the harmonic frequencies. 
great similarity of this configuration with the $L C L$ filter leads to a dependence on the sum of inductances $\left(L_{t}+L_{c}\right)$. In the case of voltage attenuation the inductance relation $L_{g} /\left(L_{t}+L_{c}\right)$ still affects the filter behavior. However, the filter performance deteriorates in the higher frequency range. The current and voltage attenuations respectively depends on the $n^{\text {th }}$ harmonic and a constant value, $\left(\left[L_{g} /\left(L_{t}+L_{c}\right)\right]\left[L_{r} /\left(L_{r}+L_{e q}\right)\right]\right.$ in this case). This resembles the first order $L$ filter. It is a classical problem of this filter configuration though the filter performance is rather superior to that of the $L$ filter.

\section{Third order LCL with Harmonic Traps.}

An alternative to the filters above consists of third order $L C L$ configuration with some resonant traps tuned in specific converter harmonics and in parallel with the main filter parallel branch. The main filter has again the elements in " $T$ " as Fig 5. The filter behavior is expressed by (17)-(20) and:

$$
\begin{gathered}
i_{f}=i_{f o}+i_{r 1}+i_{r 2}+\ldots \\
i_{f o}=C_{f} d e / d t \\
e=L_{r 1} \frac{d i_{r 1}}{d t}+\frac{1}{C_{r 1}} \int i_{r 1} d t \\
e=L_{r 2} \frac{d i_{r 2}}{d t}+\frac{1}{C_{r 2}} \int i_{r 2} d t
\end{gathered}
$$

Taking the Laplace transforms of them with resistive elements neglected the transfer functions $H_{v}(s)$ and $H_{u}(s)$ are:

$$
\begin{aligned}
& H_{v}(s)=\frac{\left\{1+s^{2} L_{c}\left[C_{f}+C_{r 1} /\left(1+s^{2} L_{r 1} C_{r 1}\right)+C_{r 2} /\left(1+s^{2} L_{r 2} C_{r 2}\right)+. .\right]\right\}}{s\left(L_{t}+L_{c}\right)\left\{1+s^{2} L_{e q}\left[C_{f}+C_{r 1} /\left(1+s^{2} L_{r 1} C_{r 1}\right)+C_{r 2} /\left(1+s^{2} L_{r 2} C_{r 2}\right)+. .\right]\right\}} \\
& H_{u}(s)=\frac{1}{s\left(L_{t}+L_{c}\right)\left\{1+s^{2} L_{e q}\left[C_{f}+C_{r 1} /\left(1+s^{2} L_{r 1} C_{r 1}\right)+C_{r 2} /\left(1+s^{2} L_{r 2} C_{r 2}\right)+. .\right\}\right\}}
\end{aligned}
$$

Replacing the Laplace operator $s$ with $j n \omega_{1}$ and splitting the filter equivalent circuit in two, at $\omega_{1}$ and $n \omega_{1}$ (with all harmonic voltage $v_{g n}$ neglected):

$i_{g 1}=\frac{\left.\left\{1-\omega_{1}^{2} L_{c} \mid C_{f}+C_{r 1} /\left(1-\omega_{1}^{2} L_{r 1} C_{r 1}\right)+C_{r 2} /\left(1-\omega_{1}^{2} L_{r 2} C_{r 2}\right)+. .\right\}\right\} v_{g 1}-u_{1}}{j \omega_{1}\left(L_{t}+L_{c}\right)\left\{1-\omega_{1}^{2} L_{e q} \mid C_{f}+C_{r 1} /\left(1-\omega_{1}^{2} L_{r 1} C_{r 1}\right)+C_{r 2} /\left(1-\omega_{1}^{2} L_{r 2} C_{r 2}\right)+. .\right\}}$
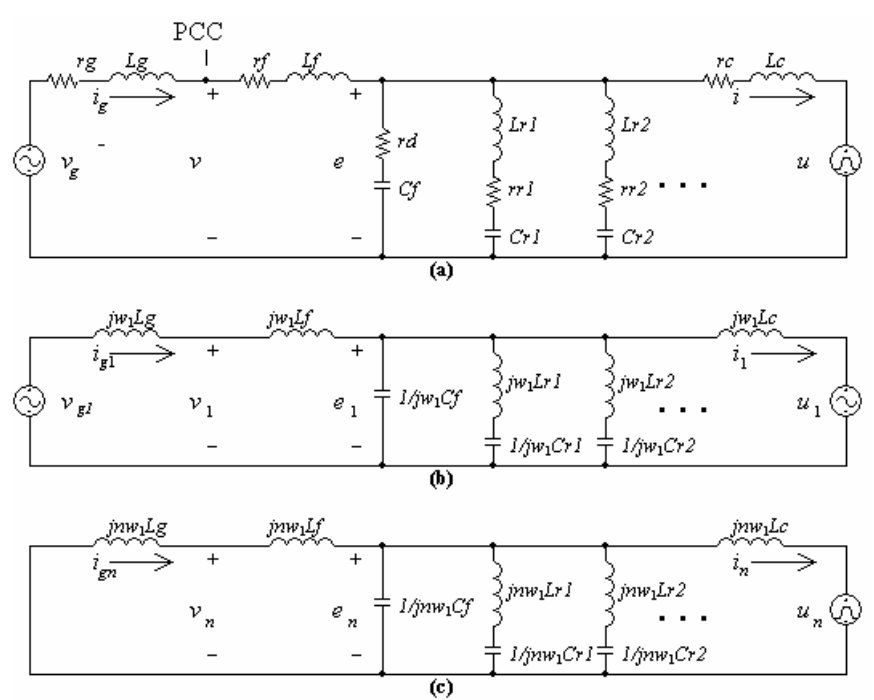

Fig. 5. System model with $L C L$ with harmonic traps. a) Single phase equivalent circuit, b) equivalent circuit for the fundamental frequency, c) equivalent circuit for the harmonic frequencies.

$$
\begin{aligned}
& i_{g n}=\frac{u_{n}}{j n \omega\left(L_{t}+L_{c}\right)\left\{1-n^{2} \omega^{2} L_{e q}\left[C_{f}+\frac{C_{r 1}}{\left(1-n^{2} \omega^{2} L_{r 1} C_{r 1}\right)}+\frac{C_{r 2}}{\left(1-n^{2} \omega^{2} L_{r 2} C_{r 2}\right)}+\ldots\right]\right\}} \\
& v_{1}=v_{g 1}-\frac{L_{g}\left\{1-\omega_{1}^{2} L_{c} \mid C_{f}+C_{r 1}\left(1-\omega_{1}^{2} L_{r 1} C_{r 1}\right)+C_{r 2} /\left(1-\omega_{1}^{2} L_{r 2} C_{r 2}\right)+. .\right] j \nu_{g 1}-L_{g} u_{1}}{\left.\left(L_{t}+L_{c}\right)\left\{1-\omega_{1}^{2} L_{e q} \mid C_{f}+C_{r 1} /\left(1-\omega_{1}^{2} L_{r 1} C_{r 1}\right)+C_{r 2} /\left(1-\omega_{1}^{2} L_{r 2} C_{r 2}\right)+. .\right]\right\}} \\
& v_{n}=\frac{L_{g}}{\left(L_{t}+L_{c}\right)} \frac{u_{n}}{\left\{1-n^{2} \omega_{1}^{2} L_{e q}\left[C_{f}+\frac{C_{r 1}}{\left(1-n^{2} \omega_{1}^{2} L_{r 1} C_{r 1}\right)}+\frac{C_{r 2}}{\left(1-n^{2} \omega_{1}^{2} L_{r 2} C_{r 2}\right)}+\ldots\right]\right\}}
\end{aligned}
$$

The rms grid current $I_{d i s}$ and voltage $V_{d i s}$ distortions are:

$$
\begin{aligned}
& I_{d i s}=\frac{1}{\omega_{1}\left(L_{t}+L_{c}\right)} \sqrt{\sum_{n=2}^{n_{m}} \frac{U_{n}^{2} / n^{2}}{\left\{1-n^{2} \omega_{1}^{2} L_{e q}\left[C_{f}+\frac{C_{r 1}}{\left(1-n^{2} \omega_{1}^{2} L_{r 1} C_{r 1}\right)}+\frac{C_{r 2}}{\left(1-n^{2} \omega_{1}^{2} L_{r 2} C_{r 2}\right)}+\ldots\right]\right\}^{2}}} \\
& V_{d i s}=\frac{L_{g}}{L_{t}+L_{c}} \sqrt{\sum_{n=2}^{n_{m=n}} \frac{U_{n}^{2}}{\left\{1-n^{2} \omega_{1}^{2} L_{e q}\left[C_{f}+\frac{C_{r 1}}{\left(1-n^{2} \omega_{1}^{2} L_{r 1} C_{r 1}\right)}+\frac{C_{r 2}}{\left(1-n^{2} \omega_{1}^{2} L_{r 2} C_{r 2}\right)}+\ldots\right]\right\}^{2}}}
\end{aligned}
$$

The analysis shows that the current and voltage harmonics depend among others on the product $n^{2} \omega_{1}^{2} L_{e q}\left[C_{f}+\sum_{k=1}^{n r r} C_{r k} /\left(1-n^{2} \omega_{1}^{2} L_{r k} C_{r k}\right)\right]$, (nrr is the number of parallel resonant branches adopted in the filter design). The equations are undoubtedly more elaborated than that of $L C L ' L$ filter. This gives great flexibility to the filter design, [4]. The parallel branches add the capacitive terms $C_{r k} /\left(1-n^{2} \omega_{1}^{2} L_{r k} C_{r k}\right) \quad\{k=1,2, \ldots, n r r\}$, to the main filter capacitor $C_{f}$ in such a way that for the lower order harmonics $C_{f}+\sum C_{r k} /\left(1-n^{2} \omega_{1}^{2} L_{r k} C_{r k}\right)$ tends to $C_{f}+\sum C_{r k}$ and for higher frequency harmonics it tends to $C_{f}$. This implies that $L C L$ filter with harmonic traps behaves like the third order $L C L$ filter in high frequency region. On the other hand, it is possible to show that the filter works as $L C L^{\prime} L$ in low frequency range. This gives the filter high attenuation ratio and selectivity along with good high frequency response.

\section{Filter Evaluation.}

Fig. 6 presents ideal (no damping and losses) filter frequency responses. Bearing in mind the current harmonic attenuation the $L$ filter exhibits a typical first order frequency response, ( $\cong-20 \mathrm{~dB} / \mathrm{dec}$.), but its voltage attenuation is poor, (constant in all frequency range). The third order $L C L$ improves the filter performance but besides resonance, its attenuation in low frequency range is not so high. The $L C L^{\prime} L$ filter enhances this feature with a resonant branch, which can be tuned in a specific low frequency harmonic. A drawback in this case is the deterioration of its high frequency response to that of a first order filter, $(-20 \mathrm{~dB} / \mathrm{dec}$ and constant for the current and voltage attenuations respectively). Instead, the Fig. 6 shows the following advantages of $L C L$ filter with harmonic traps over the other filter configurations:

- Higher harmonic attenuation ratio due to harmonic traps;

- Attenuation of two or more low frequency harmonics according to the design specifications;

- No deterioration of its high frequency response due to the independent tuned branch;

- Good performance in both high and low frequency 
regions. In the first case, it takes the advantage of $L C L$ filter behavior. In the second case, it improves $L C L^{\prime} L$ attenuation capability in low frequency range;

- Better attenuation profile among all filter configuration, which results in a good adaptation to practically all modulation techniques, (with either well defined sidedband harmonic components around multiples of switching frequency or specific non-eliminated harmonic resultant from pre-calculated pulse patterns).

Additionally, Table I compares the complexity of the filters in Fig. 6. According to [9] filter cost and size increases with its total reactive power and thus with individual values of reactive elements. Nonetheless, the improvement in filter performances justifies the increase in their complexities.

\section{SElective Harmonic EliminAtion - SHEPWM.}

The power circuits consist of a Neutral Point Clamped converter working as active rectifier and a passive sinusoidal input filter. References [7] and [8] discuss the generalized method of Selective Harmonic Elimination and state that an arbitrary number $M$ of harmonic could be controlled and/or eliminated from a standard three level output voltage
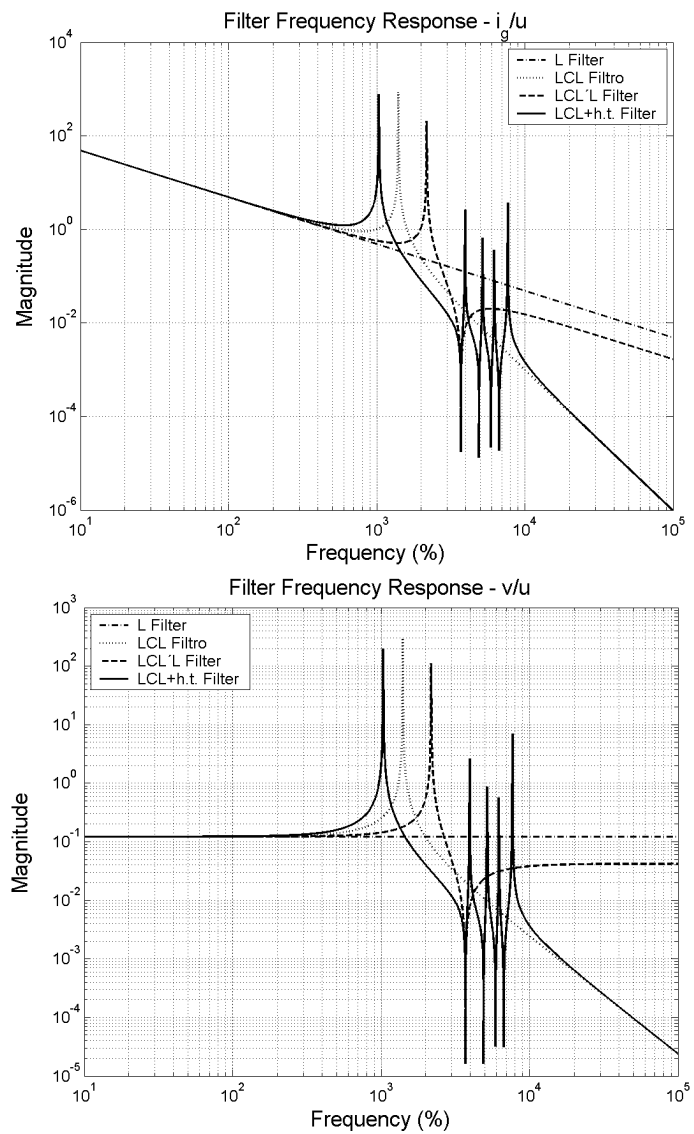

Fig. 6. Frequency response of high power filter configurations.

TABLE I

RATINGS OF ELEMENTS FOR THE FILTER CONFIGURATIONS (PER UNIT).

\begin{tabular}{|c|c|c|c|c|c|c|c|}
\hline Filter & $L_{c}$ & $L_{f}$ & $C_{f}$ & $L_{r 1}$ & $C_{r 1}$ & $L_{r 2}$ & $C_{r 2}$ \\
\hline$L$ & 0.180 & - & - & - & - & - & - \\
\hline$L C L$ & 0.100 & 0.081 & 0.100 & - & - & - & - \\
\hline$L C L ' L$ & 0.100 & 0.081 & 0.100 & 0.027 & - & - & - \\
\hline$L C L+$ ht & 0.100 & 0.081 & 0.100 & 0.027 & 0.027 & 0.020 & 0.020 \\
\hline
\end{tabular}

waveform if it is chopped $M$ times per half fundamental period. Expanding the waveform into Fourier series and assuming odd quarter-wave symmetry, the cosine components vanish and (51) gives $n^{\text {th }}$ harmonic coefficient of the sine components, $(n=1,3,5 \ldots)$.

$$
\begin{gathered}
a_{n}=(4 E / \pi n) \sum_{k=1}^{M}(-1) \cdot{ }^{k+1} \cdot \cos \left(n \alpha_{k}\right) \\
0 \leq \alpha_{1}<\alpha_{2} \cdots<\alpha_{M}<\pi / 2
\end{gathered}
$$

In order to control the fundamental component and eliminate $(M-1)$ harmonics it is essential to solve a nonlinear and transcendental system of equations. Based on the Newton's method in [7] it was developed an algorithm to solve those equations in order to control the converter fundamental component and eliminate $5^{\text {th }}$ and $7^{\text {th }}$ harmonics.

\section{RESULTS}

Fig. 7 shows the results for a three-level NPC rectifier of $5 \mathrm{MVA} / 4160 \mathrm{~V} 60 \mathrm{~Hz}$, dc bus voltage $v_{d c}=3236 \mathrm{~V}$ simulated with MATLAB/SIMULINK. SHEPWM generates switching command at frequency $f_{c}=180 \mathrm{~Hz}$. The sinusoidal main filter elements are all about $0.15 \mathrm{pu}$, (Table II). The system shortcircuits inductance $L_{g}$ is assumed equal to $0.025 \mathrm{pu}$. The rectifier is under control of voltage oriented synchronous reference frame current loop and the converter high power factor is achieved setting the reference current $i_{q}^{*}=-148 \mathrm{~A}$ by the controller. In Fig. 7a, the grid line voltage $V_{a b}$ is reasonably sinusoidal in contrast to $U_{a b}$, a typical 3-level line voltage. In this case, the former presents a $T H D=0.4 \%$ with very low harmonic content in all frequency range, Fig. 8 . The latter presents a $T H D=26.61 \%$ and negligible low frequency harmonics below $660 \mathrm{~Hz}$. In Fig. $7 \mathrm{~b}, V_{a}$ and $i_{a}$ are basically in phase. The displacement power factor $D P F \approx 1$ and distortion factor given by $D F=\left(I_{s l} / I_{s}\right)=1 /\left[(T H D)^{2}+1\right]^{1 / 2} \approx 1$. It results in system operation effectively with unity power factor $(P F=$ $D P F . D F)$. Moreover $i_{a}$ is highly sinusoidal with $T H D=$ $1.37 \%$ and all harmonic amplitudes below $0.8 \%$, as in Fig. 8 b. TABLE II

RATINGS OF SINUSOIDAL FILTER ELEMENTS.

\begin{tabular}{|c|c|c|}
\hline$L_{f}$ & $1.272 \mathrm{mH}$ & $0.1385 \mathrm{pu}$ \\
\hline$L_{c}$ & $1.375 \mathrm{mH}$ & $0.1498 \mathrm{pu}$ \\
\hline$C_{f}$ & $120 \mu \mathrm{F}$ & $0.1566 \mathrm{pu}$ \\
\hline
\end{tabular}
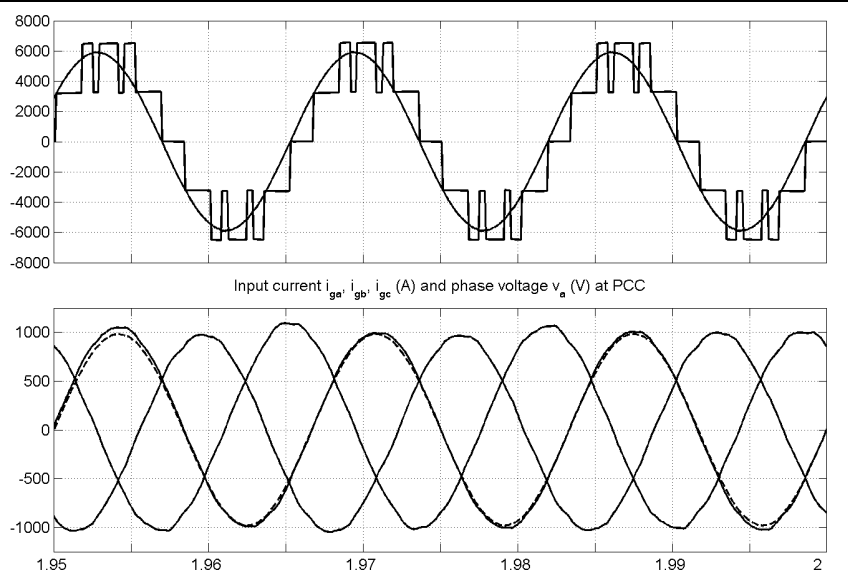

Fig. 7. a) Line grid $V_{a b}$ and converter $U_{a b}$ voltages b) grid currents $i_{a}, i_{b}, i_{c}$ and phase voltage $V_{a}$ (dashed) with sinusoidal filter. 

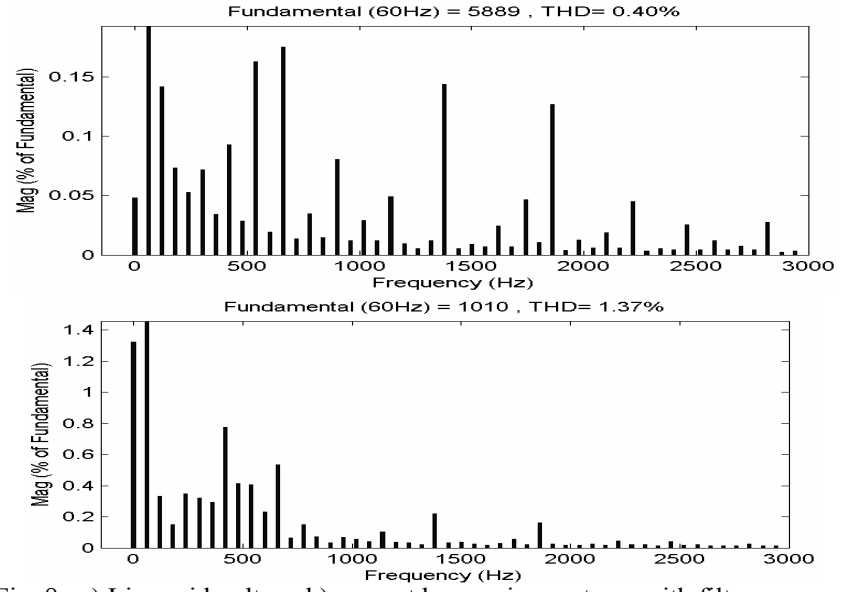

Fig. 8. a) Line grid voltage b) current harmonic spectrum with filter.

Experimental results from a lab test bench for SHEPWM and a three-level NPC converter of $10 \mathrm{kVA} / 460 \mathrm{~V} 60 \mathrm{~Hz}$ are in Fig. 9 and 10. Fig. 9 shows the converter pulse pattern of phase and line voltages. The line voltage spectrum in this case is in Fig. 10. The Table III shows the amplitude of the first 10 odd line voltage harmonics.

\section{CONCLUSIONS}

This work investigated four important filter configurations for harmonic reduction in high power rectifiers. They are the first order $L$ filter, the third order $L C L$ filter, the tuned $L C L{ }^{\prime} L$ filter and the $L C L$ filter with harmonic traps. The paper presented and analyzed their idealized mathematic equations and models and compared their frequency responses. It is remarkable that the simpler filter configuration the worse its

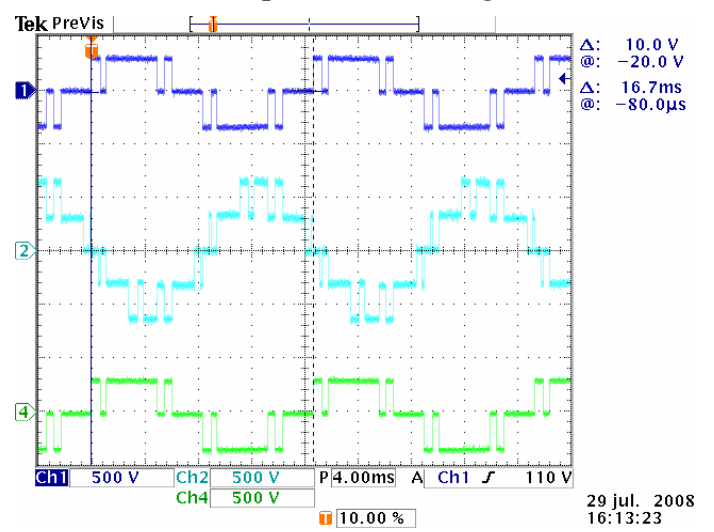

Fig. 9. SHEPWM phase and line voltages, $U_{a o}, U_{b o}$ and $U_{a b}$

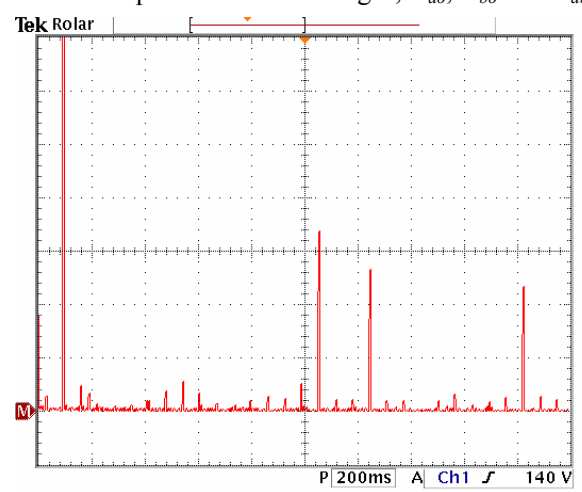

Matem. $20.0 \mathrm{~V} \quad 125 \mathrm{~Hz}$ 29 jul. 2008
TABLE III

SHEPWM-LINE VOLTAGE HARMONICS.

\begin{tabular}{|c|c|r|}
\hline$n$ & Frequency $f_{n}(\mathrm{~Hz})$ & $U_{n}(\%)$ \\
\hline $1^{\mathrm{o}}$ & 60 & 100.00000 \\
\hline $3^{\mathrm{o}}$ & 180 & 0.93443 \\
\hline $5^{\mathrm{o}}$ & 300 & 1.43095 \\
\hline $7^{\mathrm{o}}$ & 420 & 0.56376 \\
\hline $9^{\mathbf{0}}$ & 540 & 0.25467 \\
\hline $11^{\mathrm{o}}$ & 660 & 17.17404 \\
\hline $13^{\circ}$ & 780 & 14.85169 \\
\hline $15^{\circ}$ & 900 & 1.03137 \\
\hline $17^{\mathrm{o}}$ & 1020 & 0.63141 \\
\hline $19^{\circ}$ & 1140 & 14.75186 \\
\hline
\end{tabular}

performance is. This results in less flexibility of filter design and perhaps in heavier filter elements to suit some design specification. Oppositely, the $L C L$ filter with harmonic traps is clearly more elaborated than the other filters and for this motive, it shows outstanding features and performance. Besides it presents superior attenuation profile among all filter configuration, which results in a good adaptation to practically all modulation techniques, (with either well defined sided-band harmonic components around multiples of switching frequency or specific non-eliminated harmonic resultant from pre-calculated pulse patterns). Along with it the work takes advantage of Selective Harmonic Elimination which is an important modulation technique considering the converter limited switching frequency and filter bandwidth. It makes feasible the filter design in low frequency conditions due to its larger frequency range free of harmonics. Simulation of a high power rectifier demonstrated that it is possible synthesize sinusoidal voltage and current with acceptable operation performance under low switching frequency, even with a reduced level structure such as the three-level NPC converter.

\section{REFERENCES}

[1] S. B. Dewan and E. B. Shahrodi, "Design of an input filter for the sixpulse bridge rectifier," IEEE Trans. Industry Applications, vol. IA-21, pp. 1168-1175, Sep./Oct. 1985.

[2] N. R. Zargari, G. Joos, .P. D. Ziogas "Input Filter Design for PWM Current -Source Rectifiers" IEEE Transactions on Industry Applications, Vol. 30, No. 6, pp. 1573-1579, Nov./Dec. 1994.

[3] V. Blasko, V. Kaura, "A novel control to actively damp resonance in input LC filter of a three-phase voltage source converter", IEEE Trans. on Ind. Applications, Vol. 33, No. 2, 1997, pp. 542-550.

[4] Skibinski, G.L.; "A series resonant sinewave output filter for PWM VSI loads" Industry Applications Conference, 2002. 37th IAS Annual Meeting. Conference Record of the vol. 1, 13-18 Oct. 2002 pp:247-256.

[5] Mohan, N; Underland, T.M.; Robbins, W. P.; "Power Electronics, converters, applications and design", John Wiley \& Sons, USA, 1989.

[6] Liserre, M.; Blaabjerg, F.; Hansen, S.; "Design and control of an LCLfilter-based three-phase active rectifier" IEEE Transactions on Industry Applications, Vol. 41, No. 5, Sept.-Oct. 2005 pp.: $1281-1291$.

[7] H. S. Patel and R. G. Hoft, "Generalized techniques of harmonic elimination and voltage control in thyristor inverters: Part II-Voltage control techniques," IEEE Trans. Ind. Applicat., vol. IA-10, no. 5, pp. 666-673, Sept./Oct. 1974.

[8] L.G. Franquelo; J. Napoles; R.C.P. Guisado; J.I. Leon; M.A. Aguirre, "A Flexible Selective Harmonic Mitigation Technique to Meet Grid Codes in Three-Level PWM Converters", IEEE Trans. on Ind. Electronics, Vol. 54, No. 6, 2007, pp. 3022-3029.

[9] H-s. Kim J-h. Choi "Input Filter Design for Thyristor Converter with LCL' Configuration" Conference Proceedings, Budapest, IEEE International Symposium on Industrial Electronics, 1993, pp.599-604. 